\title{
Demographics of severe valvular aortic stenosis in Singapore
}

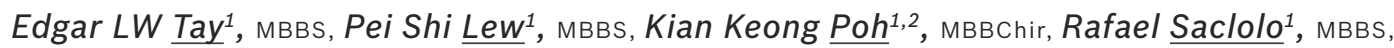

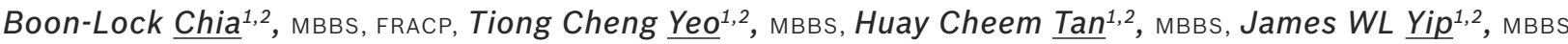

INTRODUCTION The treatment of aortic valve stenosis (AS) is seeing renewed interest mainly due to the availability of transcatheter therapies. However, the number of epidemiological studies of this disease in Singapore is limited. We aimed to describe the aetiology and clinical presentation of AS in Singapore, as well as patients' attitudes toward it. Our findings may facilitate the future planning and utilisation of resources to better manage these patients.

METHODS 249 consecutive patients who underwent transthoracic echocardiography (from April 1999 to April 2008) and diagnosed with severe AS were assessed. Demographic and clinical data were collected, and patients' decisions on surgery were determined.

RESULTS The mean patient age was 71 (range 23-98) years. 50.2\% of patients were male. The commonest presenting symptom was dyspnoea, and $40(16.0 \%)$ patients had coexistent atrial fibrillation. The aetiology of AS was degenerative in $216(86.7 \%)$, rheumatic in $11(4.4 \%)$ and related to a bicuspid valve in $22(8.9 \%)$ patients. The average peak velocity across the aortic valve was $4.2 \pm 0.8 \mathrm{~m} / \mathrm{s}$ and the mean aortic valve area was $0.76 \pm 0.13 \mathrm{~cm}^{2}$. The overall mean logistic EuroSCORE was $10.7 \pm 12.3 .105(42.2 \%)$ patients who were offered surgery refused. $87(35 \%)$ deaths were seen during the follow-up period (mean duration 14.5 months), which also saw 68 (27\%) patients undergo surgery and 86 (34\%) patients hospitalised for heart failure.

CONCLUSION Degenerative AS was the commonest aetiology in this contemporary cohort of patients. Despite the known benefits of surgery, the refusal rate for surgery remained high.

Keywords: epidemiology, severe aortic stenosis, transcatheter aortic valve implantation

singapore Med J 2013; 54(1): 36-39

\section{INTRODUCTION}

With the increasing proportion of elderly patients in Singapore, the incidence of severe aortic valve stenosis (AS) (degenerative) is likely to increase. However, there is currently little published data on the epidemiology of AS in the country. We aimed to describe the aetiology and clinical presentation of AS in Singapore, as well as determine patients' attitudes toward it.

This study is important for a few reasons. Firstly, many patients with severe AS, especially the elderly, would have associated comorbidities. ${ }^{(1)}$ Although studies have demonstrated benefits from surgical aortic valve replacement, ${ }^{(2-6)}$ such comorbidities are likely to have an impact on associated risks, complications and the length of hospital stay in this cohort of patients. For this reason, better planning and provision of resources would be possible once the extent of such comorbidities is established. Secondly, patients who are deemed inoperable can now benefit from transcatheter valve therapies. ${ }^{(7,8)}$ The currently available trancatheter heart valves are suitable for aortic annuli of between 18-27 mm. ${ }^{(9)}$ The dimensions of aortic annuli in Asians with AS have not been well studied, and the proportion of patients who may be suitable for these procedures is still unknown. Thirdly, the attitudes of patients and physicians toward AS are largely unknown. According to some studies, up to one-third of patients who have severe symptomatic AS are untreated. ${ }^{(10,11)}$ Therefore, it becomes important to determine the reasons for the refusal of treatment, as quality of life and longevity can be improved with successful therapy in patients with AS..$^{(6,12)}$

\section{METHODS}

This study commenced after obtaining ethics approval from the institutional review board. Patients who had undergone transthoracic echocardiography (TTE) at the National University Hospital, Singapore, between April 1999 and April 2008 and were diagnosed with severe AS were selected for enrolment. The clinical and demographic characteristics of patients were accessed from paper and electronic clinical records as well as from the hospital's echocardiographic database.

The diagnosis of AS was made according to the American College of Cardiology guidelines, ${ }^{(13)}$ and the criteria included: two-dimensional features of limited valve excursion with a Doppler-derived maximum jet velocity across the aortic valve of $4 \mathrm{~m} / \mathrm{s}$, a mean gradient $>40 \mathrm{mmHg}$ or an estimated aortic valve area of $<1 \mathrm{~cm}^{2}$ (by planimetry or continuity equation). The aortic annulus, sinus, sinotubular junction and the ascending aorta diameters were measured by standard two-dimensional techniques from the parasternal long axis views. Besides these variables, other information collected included comorbidities such as smoking, diabetes mellitus, hyperlipidaemia and hypertension. The logistic EuroSCORE, ${ }^{(14,15)}$ which assesses the risk of surgery at the point of diagnosis, was also calculated. The patients' most

${ }^{1}$ National University Heart Centre, National University Health System, ${ }^{2}$ Department of Medicine, Yong Loo Lin School of Medicine, National University of Singapore, Singapore

Correspondence: Dr Edgar Tay, Consultant Cardiologist, National University Heart Centre, 1E Kent Ridge Road, NUHS Tower Block, Level 9, Singapore 119228. Edgar_Tay@nuhs.edu.sg 
current medications were recorded. Clinical outcomes that were assessed (via phone call, review of case notes, electronic in-hospital and island-wide medical records) included death, surgical valve replacement and first hospitalisation for heart failure. In addition, the patient's final decision on treatment type after being offered surgery was assessed.

Baseline characteristics were summarised into frequencies and percentages for categorical variables and as mean \pm standard deviation for continuous variables. The characteristics of patients with different aetiologies of AS were determined. Continuous variables were tested for normal distribution using the Kolmogorov-Smirnov test and compared with one-way analysis of variance (ANOVA). Categorical variables were compared using chi-square and Fisher's exact tests. Data was analysed using the Statistical Package for the Social Sciences for Windows version 15 (SPSS Inc, Chicago, IL, USA).

\section{RESULTS}

A total of 249 patients were diagnosed with severe AS during the study period. The most common cause of AS was degenerative calcific AS ( $n=216 ; 86.7 \%)$, followed by bicuspid valve $(n=22$; $8.9 \%$ ) and rheumatic AS ( $\mathrm{n}=11 ; 4.4 \%$ ) (Fig. 1 and Table I). The mean peak velocity across the aortic valve was $4.2 \pm 0.8 \mathrm{~m} / \mathrm{s}$ and the mean aortic valve area was $0.76 \pm 0.13 \mathrm{~cm}^{2}$. Not surprisingly, patients with bicuspid AS were significantly younger than those with a degenerative or rheumatic aetiology (62 years vs. 72 years; $p<0.001)$. Comorbidities such as hypertension were more prevalent in patients with degenerative AS (58.7\%) compared to those with a rheumatic $(36.4 \%)$ or bicuspid $(36.4 \%)$ aetiology (Table I).

Asymptomatic AS was common (47.0\%). Among the symptomatic patients, dyspnoea $(31.7 \%$ ) was the most frequent presentation, followed by chest pain (8.4\%) and acute coronary syndrome (6.4\%). Syncope was infrequent (3.6\%). A significant proportion of patients had concomitant atrial fibrillation in the rheumatic group $(54.5 \%)$ compared to the other two groups (degenerative $14.4 \%$, bicuspid $13.6 \%$; $p=0.004$ ). Among the patients in the rheumatic group with atrial fibrillation, all had concomitant moderate grade or more mitral stenosis and three had severe mitral regurgitation. Coexisting ischaemic heart disease diagnosed on coronary angiography was common (40.0\%).

The mean logistic EuroSCORE was higher in patients with degenerative AS $(11.5 \pm 12.7 ; p=0.085)$. Interestingly, the rates of patients who declined surgery were very high in our series, with $42 \%, 36 \%$ and $46 \%$ of patients with degenerative, rheumatic and bicuspid AS, respectively, refusing surgical treatment. The median follow-up period was 14.5 months, with 11 patients lost to follow-up. The rates of death ( $\mathrm{n}=87,35 \%$ ), surgery $(n=68,27 \%)$ and hospitalisation due to heart failure $(n=86,34 \%)$ among patients during the follow-up period were high. 29 of the 117 asymptomatic patients (25\%) died on follow-up compared to 58 of the 132 symptomatic patients $(44 \%)$ [p $=0.02$ ]. $23(20 \%)$ asymptomatic patients were hospitalised compared to 63 (47\%)

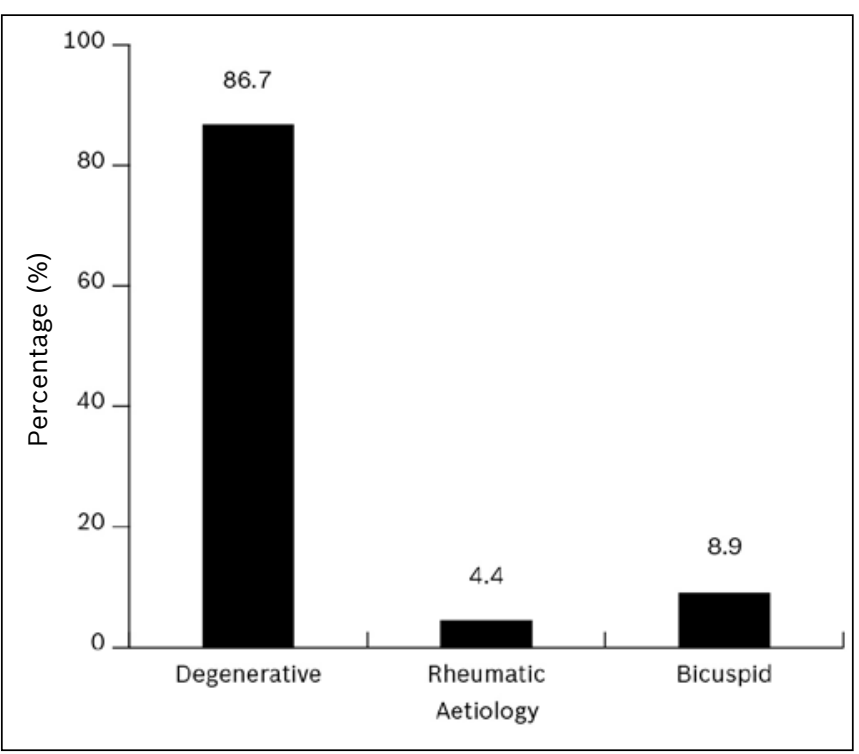

Fig. 1 Bar graph shows the aetiology of aortic valve stenosis among the study patients.

symptomatic patients $(p<0.01)$. Echocardiographic evaluations of the aortic dimension showed a mean annulus size of $20.3 \pm$ $2.0 \mathrm{~mm}$ (Table II). 15 patients had an aortic annulus $<18 \mathrm{~mm}$, of whom five patients had an annulus size of $16 \mathrm{~mm}$ and ten patients had an annulus size of $17 \mathrm{~mm}$. All except for one among these 15 patients were women. None of the patients had an aortic annulus exceeding $27 \mathrm{~mm}$.

\section{DISCUSSION}

This was a contemporary study of the epidemiology of severe AS in Singapore. It highlights the many unique features of Asian patients. With regard to the symptoms of $\mathrm{AS}$, the most frequent in our series was dyspnoea, with a large proportion of patients also presenting with concomitant atrial fibrillation. Several patients presented with a first episode of heart failure with new onset atrial fibrillation (16.0\%). This was not surprising given the age of the cohort. There were more atrial fibrillation episodes in patients with rheumatic AS, as many of these patients had coexistent mitral valve disease.

The logistic EuroSCORE, which is often used as a surrogate marker of preoperative risk for patients with AS, has also been used for the selection of patients for transcatheter aortic valve implantation (TAVI). ${ }^{(16)}$ The mean logistic EuroSCORE of 10.7 \pm 12.3 , seen in our series, was not reflective of a very high-risk cohort, as most transcatheter valve selection criteria would select patients with logistic EuroSCORE $>20$. In spite of these moderate risk scores, it was interesting to find that many of our patients refused surgery. Other studies on AS have similarly reported a high rate of patients opting not to undergo surgery despite having adequate surgical indications. ${ }^{(10)}$ We were unable to determine the reasons for patients refusing surgery in our study. This should be the focus of future efforts.

The event rates in our study were very high, which is consistent with other published studies of severe AS. ${ }^{(17,18)}$ Although our cohort had both symptomatic and asymptomatic patients, whether all of 
Table I. Characteristics of patients with aortic stenosis.

\begin{tabular}{|c|c|c|c|c|c|}
\hline \multirow[t]{2}{*}{ Variable } & \multicolumn{4}{|c|}{ Aetiology of aortic stenosis [No. (\%)] } & \multirow[t]{2}{*}{ p-value } \\
\hline & $\begin{array}{c}\text { Degenerative } \\
\text { (n= 216) }\end{array}$ & $\begin{array}{l}\text { Rheumatic } \\
\qquad(n=11)\end{array}$ & $\begin{array}{l}\text { Bicuspid } \\
(n=22)\end{array}$ & $\begin{array}{c}\text { Total } \\
(n=249)\end{array}$ & \\
\hline Mean age (range) (yrs) & $72(40-98)$ & $76.2(51-90)$ & $61.8(23-85)$ & $71(23-98)$ & $<0.001$ \\
\hline Male gender & $106(49.1)$ & $3(27.3)$ & $16(72.7)$ & $125(50.2)$ & 0.03 \\
\hline Ethnicity & & & & & 0.78 \\
\hline Malay & $45(20.8)$ & $3(27.3)$ & $6(27.3)$ & $54(21.7)$ & \\
\hline Indian & $15(7.0)$ & $0(0)$ & $2(9.1)$ & $17(6.8)$ & \\
\hline Other & $25(11.6)$ & $0(0)$ & $2(9.1)$ & $27(10.8)$ & \\
\hline Weight* (kg) & $58.6 \pm 11.4$ & $59.1 \pm 12.2$ & $59.9 \pm 12.1$ & $58.6 \pm 11.4$ & 0.85 \\
\hline Height* (cm) & $158.8 \pm 9.2$ & $159.4 \pm 9.4$ & $162.2 \pm 6.5$ & $158.5 \pm 10.0$ & 0.12 \\
\hline Body surface area* $\left(\mathrm{cm}^{2}\right)$ & $1.59 \pm 0.18$ & $1.61 \pm 0.20$ & $1.64 \pm 0.18$ & $1.60 \pm 0.18$ & 0.56 \\
\hline \multicolumn{6}{|l|}{ Comorbidity } \\
\hline Diabetes mellitus & $77(36.2)$ & $2(18.7)$ & $5(22.7)$ & $84(33.7)$ & 0.17 \\
\hline Hypertension & $125(58.7)$ & $4(36.4)$ & $8(36.4)$ & $139(55.8)$ & 0.02 \\
\hline Dyslipidaemia & 75 (35.2) & $2(18.2)$ & $5(22.7)$ & 83 (33.3) & 0.15 \\
\hline Smoking & $24(11.3)$ & $2(18.2)$ & $7(31.8)$ & $34(13.7)$ & 0.04 \\
\hline Family history of IHD & $2(0.9)$ & $0(0)$ & $0(0)$ & $2(0.8)$ & 0.85 \\
\hline Presenting symptom & & & & & 0.03 \\
\hline Dyspnoea & 69 (31.9) & $7(63.6)$ & $3(13.6)$ & $79(31.7)$ & \\
\hline Chest pain & $18(8.3)$ & $0(0)$ & $3(13.6)$ & $21(8.4)$ & \\
\hline Syncope & $6(2.8)$ & $0(0)$ & $3(13.6)$ & $9(3.6)$ & \\
\hline Acute coronary syndrome & $16(7.4)$ & $0(0)$ & $0(0)$ & $16(6.4)$ & \\
\hline Asymptomatic & $107(50.0)$ & $4(36.3)$ & $13(59.1)$ & $124(49.8)$ & \\
\hline Atrial fibrillation & $31(14.4)$ & $6(54.5)$ & $3(13.6)$ & $40(16.0)$ & $<0.01$ \\
\hline \multicolumn{6}{|l|}{ Other findings } \\
\hline Ejection fraction* (\%) & $53.4 \pm 17.4$ & $57.8 \pm 16.3$ & $62.4 \pm 14.0$ & $54.4 \pm 17.2$ & 0.05 \\
\hline EuroSCORE* & $11.5 \pm 12.7$ & $8.0 \pm 6.7$ & $5.1 \pm 9.1$ & $10.7 \pm 12.3$ & 0.09 \\
\hline Coexisting IHD & $82(37.9)$ & $4(36.3)$ & $11(50.0)$ & $97(40.0)$ & 0.14 \\
\hline \multicolumn{6}{|l|}{ Medication } \\
\hline Aspirin & $104(48.1)$ & $3(27.0)$ & $7(31.8)$ & $114(67.5)$ & 0.11 \\
\hline Statin & $91(42.0)$ & $1(9.0)$ & $7(31.8)$ & $99(47.1)$ & 0.02 \\
\hline ACE inhibitor & $76(35.2)$ & $1(9.0)$ & $11(50.0)$ & $88(42.1)$ & 0.06 \\
\hline$\beta$-Blockers & $82(37.9)$ & $5(45.5)$ & $7(31.8)$ & $94(45.0)$ & 0.62 \\
\hline Patient consent to surgery & & & & & 0.40 \\
\hline Consented & $58(26.9)$ & $6(54.5)$ & $9(41.0)$ & 73 (29.3) & \\
\hline Refused & $91(42.1)$ & 4 (36.3) & $10(45.5)$ & $105(42.2)$ & \\
\hline Not offered & $23(10.6)$ & $0(0)$ & $2(9.1)$ & $25(10.0)$ & \\
\hline Uncertain & $44(20.4)$ & $1(9.1)$ & $1(4.5)$ & $46(18.5)$ & \\
\hline
\end{tabular}

*Data is presented as mean \pm standard deviation.

BMI: body mass index; IHD: ischaemic heart disease; ACE: angiotensin-converting enzyme

Table II. Echocardiographic findings of patients with aortic stenosis.

\begin{tabular}{|c|c|c|c|c|c|}
\hline \multirow[t]{2}{*}{ Aortic dimension } & \multicolumn{4}{|c|}{ Aetiology of aortic stenosis [Mean \pm SD] } & \multirow[t]{2}{*}{ p-value } \\
\hline & $\begin{array}{c}\text { Degenerative } \\
(n=216)\end{array}$ & $\begin{array}{c}\text { Rheumatic } \\
(n=11)\end{array}$ & $\begin{array}{l}\text { Bicuspid } \\
(n=22)\end{array}$ & $\begin{array}{c}\text { Total } \\
(n=249)\end{array}$ & \\
\hline Annulus (mm) & $20.2 \pm 1.9$ & $20.7 \pm 1.3$ & $21.2 \pm 2.5$ & $20.3 \pm 2.0$ & 0.045 \\
\hline Sinus $(\mathrm{mm})$ & $32.3 \pm 5.0$ & $30.5 \pm 1.4$ & $33.1 \pm 4.8$ & $32.3 \pm 5.0$ & 0.671 \\
\hline Sinotubular junction ( $\mathrm{mm}$ ) & $26.5 \pm 5.4$ & $24.7 \pm 2.1$ & $27.9 \pm 3.6$ & $26.6 \pm 5.2$ & 0.501 \\
\hline Ascending aorta (mm) & $33.6 \pm 6.0$ & $30.2 \pm 4.2$ & $36.4 \pm 4.6$ & $33.9 \pm 5.9$ & 0.114 \\
\hline Upper ascending aorta (mm) & $35.4 \pm 6.2$ & $33.1 \pm 2.96$ & $35.3 \pm 6.0$ & $35.3 \pm 6.0$ & 0.875 \\
\hline
\end{tabular}

SD: standard deviation

our asymptomatic patients were truly asymptomatic is open to speculation, given that the assessment of symptoms in patients with AS is known to be difficult.

Our study findings hold important implications for the future management of patients with severe AS in Singapore, especially with the introduction of transcatheter techniques. Firstly, the aetiology of AS was largely due to degenerative calcific change in our series, which is consistent with studies from other developed countries. ${ }^{(6,17)}$ This subset of patients with AS is most suitable for TAVI. Rheumatic AS rates were low, but a significant number of 
patients in our cohort had bicuspid AS. The latter two aetiologies can be treated using transcatheter therapy, albeit less effectively. ${ }^{(19)}$ Secondly, the sizes of our patients' aortic annulus were smaller than those reported in other published series. ${ }^{(20)}$ This could impact the development and selection of appropriate device sizes for Asian patients. Currently, aortic annular sizes $<18 \mathrm{~mm}$ and $<19 \mathrm{~mm}$ are contraindications for TAVI using balloon expandable and self-expanding valves. Based on the above criteria, 15 (6\%) of our patients would have been deemed technically ineligible for such devices. Fortunately, most of these patients would require a transoesophageal echocardiogram to verify the annulus and might still be deemed suitable after the evaluation. Thirdly, the attitudes of patients toward major cardiac surgery remain ambivalent, as surgical treatment was refused by several of our patients despite favourable risk indications. Whether a less invasive technique would have appealed more to this group of patients remains uncertain.

The authors acknowledge several limitations in this study. The number of patients with severe AS recruited was small, as this was a single-centre study. We were unable to further explore the reasons for refusal of surgery by many of our patients, and therefore suggest that future studies should assess this dynamic in greater detail. Moreover, our median follow-up period was a modest 14.5 months. Finally, the use of TTE for the measurement of aortic dimensions may be less accurate than that of transoesophageal echocardiography and multislice computed tomography. However, TTE remains an important first-line imaging tool for patients with severe AS.

In conclusion, degenerative AS is the commonest cause of AS in Singapore, especially among elderly patients. Despite the high mortality rate associated with severe AS, a significant number of patients still refuse surgical interventions.

\section{REFERENCES}

1. Tseng EE, LeeCA, Cameron DE, etal. Aortic valve replacement in the elderly. Risk factors and long-term results. Ann Surg 1997; 225:793-802.

2. Antunes MJ. Aortic stenosis in the elderly: Rethinking strategies. Eur J Cardiothorac Surg 2006; 30:713-5.

3. Bouma BJ, van Den Brink RB, van Der Meulen JH, et al. To operate or not on elderly patients with aortic stenosis: the decision and its consequences.
Heart 1999; 82:143-8

4. Descoutures F, Himbert D, Lepage L, et al. Contemporary surgical or percutaneous management of severe aortic stenosis in the elderly. Eur Heart J 2008; 29:1410-7.

5. lung B. Management of the elderly patient with aortic stenosis. Heart 2008 ; 94:519-24.

6. Varadarajan P, Kapoor N, Bansal RC, Pai RG. Survival in elderly patients with severe aortic stenosis is dramatically improved by aortic valve replacement: Results from a cohort of 277 patients aged $>$ or $=80$ years. Eur $\mathrm{J}$ Cardiothorac Surg 2006; 30:722-7.

7. Grube E, Schuler G, Buellesfeld L, et al. Percutaneous aortic valve replacement for severe aortic stenosis in high-risk patients using the secondand current third-generation self-expanding CoreValve prosthesis: device success and 30-day clinical outcome. J Am Coll Cardiol 2007; 50:69-76.

8. Leon MB, Smith CR, Mack M, et al. Transcatheter aortic-valve implantation for aortic stenosis in patients who cannot undergo surgery. N Engl J Med 2010; 363:1597-607.

9. Bleiziffer S, Ruge H, Mazzitelli D, et al. Results of percutaneous and transapical transcatheter aortic valve implantation performed by a surgical team. Eur J Cardiothorac Surg 2009; 35:615-20.

10. Bach DS, Siao D, Girard SE, et al. Evaluation of patients with severe symptomatic aortic stenosis who do not undergo aortic valve replacement: the potential role of subjectively overestimated operative risk. Circ Cardiovasc Qual Outcomes 2009; 2:533-9.

11. Iung B, Cachier A, Baron G, et al. Decision-making in elderly patients with severe aortic stenosis: why are so many denied surgery? Eur Heart J 2005; 26:2714-20.

12. Rumsfeld JS. Valve surgery in the elderly: question of quality (of life)? J Am Coll Cardiol 2003; 42:1215-7.

13. Baumgartner $\mathrm{H}$, Hung J, Bermejo J, et al. Echocardiographic assessment of valve stenosis: EAE/ASE recommendations for clinical practice. J Am Soc Echocardiogr 2009; 22:1-23.

14. Nashef SA, Roques F, Michel P, et al. European system for cardiac operative risk evaluation (EuroSCORE). Eur J Cardiothorac Surg 1999; 16:9-13.

15. Zingone B, Pappalardo A, Dreas L. Logistic versus additive EuroSCORE. A comparative assessment of the two models in an independent population sample. Eur J Cardiothorac Surg 2004; 26:1134-40.

16. Piazza N, Wenaweser P, van Gameren $M$, et al. Relationship between the logistic EuroSCORE and the Society of Thoracic Surgeons Predicted Risk of Mortality score in patients implanted with the CoreValve ReValving system--a Bern-Rotterdam Study. Am Heart J 2010; 159:323-9.

17. Rosenhek R, Zilberszac R, Schemper M, et al. Natural history of very severe aortic stenosis. Circulation 2010; 121:151-6.

18. Varadarajan P, Kapoor N, Bansal RC, Pai RG. Clinical profile and natural history of 453 nonsurgically managed patients with severe aortic stenosis. Ann Thorac Surg 2006; 82:2111-5.

19. Wijesinghe N, Ye J, Rodés-Cabau J, et al. Transcatheter aortic valve implantation in patients with bicuspid aortic valve stenosis. JACC Cardiovasc Interv 2010; 3:1122-5.

20. Webb JG, Chandavimol M, Thompson CR, et al. Percutaneous aortic valve implantation retrograde from the femoral artery. Circulation 2006; 113:842-50. 